\title{
Recent experiments at NovoFEL user stations
}

B. A. Knyazev ${ }^{1,2}$, I. A. Azarov ${ }^{3}$, E. N. Chesnokov ${ }^{4}$, Yu. Yu. Choporova, ${ }^{1,2}$, V. V. Gerasimov ${ }^{1,2}$, Ya. I. Gorbachev ${ }^{1}$, Ya. V. Getmanov ${ }^{1,2}$, B. G. Goldenberg ${ }^{1}$, O. E. Kameshkov ${ }^{1,2}$, P. V. Koshlyakov ${ }^{4}$, I. A. Kotelnikov ${ }^{1,2}$, A. S. Kozlov ${ }^{4}$, V. V. Kubarev ${ }^{1,2}$, G. N. Kulipanov ${ }^{1}$, S. B. Malyshkin ${ }^{4}$, A. K. Nikitin ${ }^{2,5}$, P. A. Nikitin ${ }^{6}$, N. D. Osintseva ${ }^{1}$, V. S. Pavelyev ${ }^{1,7}$, S. E. Peltek ${ }^{8}$, A. K. Petrov ${ }^{4}$, V. M. Popik ${ }^{1}$, T. V. Salikova ${ }^{1}$, M. A. Scheglov ${ }^{1}$, S. S. Seredniakov ${ }^{1}$, V. N. Shastin ${ }^{9}$, O. A. Shevchenko ${ }^{1}$, V. A. Shvets ${ }^{1,2}$, D. A. Skorokhod ${ }^{1}$, A. N. Skrinsky ${ }^{1}$, S. L. Veber $^{10}$, N. A. Vinokurov ${ }^{1,2}$, V. B. Voloshinov ${ }^{6}$, R. Kh. Zhukavin 9

${ }^{1}$ Budker Institute of Nuclear Physics, Siberian Branch of Russian Academy of Sciences, Novosibirsk, Russia, b.a.knyazev@inp.nsk.su

${ }^{2}$ Novosibirsk State University, Novosibirsk, Russia

${ }^{3}$ Rzhanov Institute of Semiconductor Physics, Siberian Branch of Russian Academy of Sciences, Novosibirsk, Russia ${ }^{4}$ Voevodsky Institute of Chemical Kinetics and Combustion, Siberian Branch of Russian Academy of Sciences, Novosibirsk, Russia

${ }^{5}$ Scientific and Technological Center of Unique Instrumentation, Russian Academy of Sciences, Moscow, Russia ${ }^{6}$ Lomonosov Moscow State University, Moscow, Russia

${ }^{7}$ Samara State University, Samara, Russia

${ }^{8}$ Institute of Cytology and Genetics, Siberian Branch of Russian Academy of Sciences, Novosibirsk, Russia ${ }^{9}$ Institute for Physics of Microstructures, Russian Academy of Sciences, Nizhny Novgorod, Russia

${ }^{10}$ International Tomography Center, Siberian Branch of Russian Academy of Sciences, Novosibirsk, Russia

The Novosibirsk free-electron laser (NovoFEL) is a source of monochromatic frequency-tunable radiation [1]. This facility belongs to the Siberian Synchrotron and Terahertz Radiation Center, which is open for Russian and foreign users. The NovoFEL consists of three laser oscillators, the supposed generation regions of which cover three regions of the spectrum, shown in Fig. 1 (a) with light rectangles. The spectral ranges of generation currently available to users are marked with darker rectangles. The first laser system of the facility, generating terahertz radiation in the range of $90-240 \mu \mathrm{m}$, was commissioned in 2003, and most studies performed by users to date relates to this range. Experiments using radiation of $40-50 \mu \mathrm{m}$ have been started recently. The studies that had been performed at the first six workstations by 2015 are described in the review [2]. Since then, the facility was signifi-

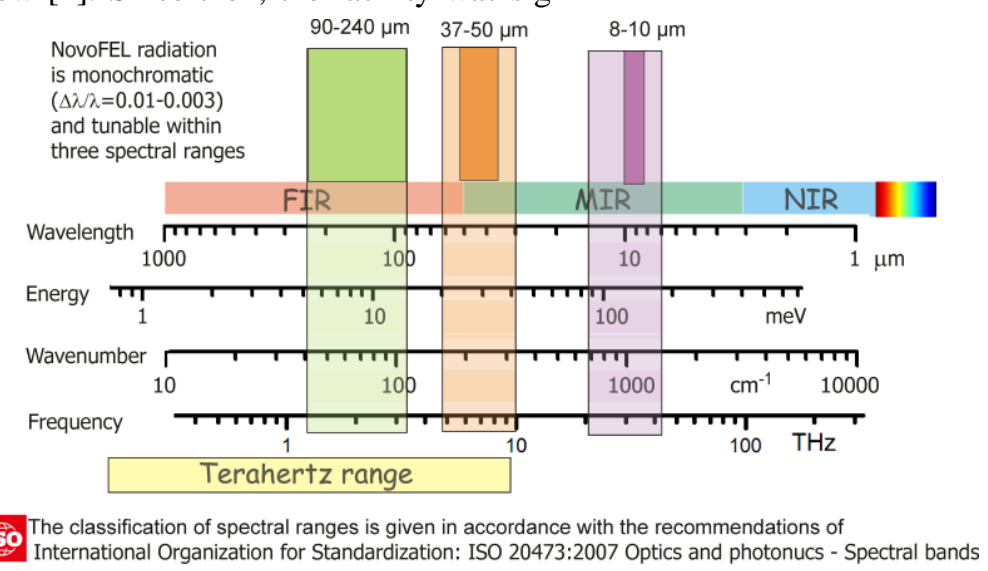

(a) cantly upgraded, and now 11 workstations, the location of which is shown in Fig. 2, are in operation. Several more stations are under construction. The directions of research conducted at the stations are understandable from their names, but are not limited by them.

The laser beams at the inputs to the user stations are Gaussian beams (Fig. 1(b)). The radiation is an infinite sequence of 30-100 ps pulses with a standard repetition rate of $5.6 \mathrm{MHz}$ and average power of up to $100-200 \mathrm{~W}$. Regimes in which the radiation is coherent are described in [3]. Such parameters enabled the development of several superfast and highresolution techniques of molecular spectroscopy [4,5], and the high pulse power allowed ignition of a continuous optical discharge in gases at the atmospheric pressure [6].

Fig. 1. (a) Generation ranges of the Novosibirsk free electron laser; (b) beam shapes at the inputs to workstations $(L$ is the distance from the laser oscillator). 


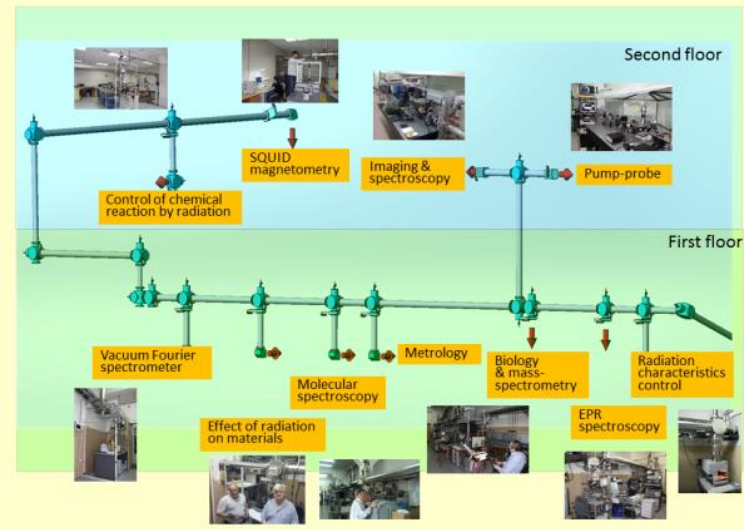

Fig. 2. Beamline (filled with dry nitrogen) and workstations at the Novosibirsk free electron laser facility.

Many applications require transformation of a Gaussian laser beam into beams of different mode structure or concentration of radiation in a predetermined volume or on an area. A number of diffractive optical elements made of diamond or high-resistivity silicon have been designed and fabricated for this purpose [7-8]. In particular, using diffractive optics made it possible to transform the laser radiation into Bessel beams with an orbital angular momentum ("vortex beams") [9-10]. The latter were converted into arrays of vortex beams via their diffraction on a 2D amplitude array of circular openings [11]. Acousto-optical deflection of a terahertz vortex beam was demonstrated in [12], and production of nanosized metal particles by acoustic waves generated in liquids by the NovoFEL pulsed-intermittent radiation was described in [13].

Classical holography with a monochromatic terahertz radiation source was first demonstrated in [14]. The NovoFEL radiation enabled achievement of record resolution for the terahertz range in the internal reflection ellipsometry [15]. Studies of terahertz surface plasmon polaritons on metal-dielectric-air interfaces revealed their particularities in comparison with the visible range plasmons [18-20].

The effect of $\mathrm{THz}$ radiation on biological objects, from cells to organisms, is under investigation at the biological station of NovoFEL (see, e. g., [21]). Two new workstations have been commissioned at the facility. A one-color pump-probe setup was applied to research on the relaxation time in semiconductors with shallow donors at cryogenic temperatures [22]. The electron paramagnetic resonance station [23] enables exploration of the influence of high-power $\mathrm{THz}$ or mid-IR radiation on spin systems.

The study was supported by the Russian Science Foundation (grant 14-50-00080). The experiments were carried out at the collective research center supported by the Ministry of Education and Science of the Russian Federation (project RFMEFI62117X0012).

\section{References}

1. Shevchenko O. A. et al. Novosibirsk free electron laser facility // These Proceedings, 2018.
2. Kulipanov G. $\mathrm{N}$ et al. Novosibirsk free electron laser as a user facility // IEEE Trans. on THz Sci. Techn. 2015. V. 5, No. 5. P. 798-809.

3. Kubarev, V. V. Instabilities, coherency, and spectra of the NovoFEL radiation // EPJ Web of Conf. 2017. V. 149. 05007.

4. Chesnokov, E. N., et al. The influence of magnetic field on the echo-like free induction decay in $\mathrm{NO}_{2} / /$ Chem. Phys. Lett. 2016. V. 662. P. 62-66.

5. Chesnokov, et al. Heterodyne Method of Detection of Molecular Gas in the Terahertz Region Using the Beats Between Free Induction Decay Signals // IEEE Trans. on THz Sci. Techn. 2017. V. 7, No. 2. P. 144-150.

6. Kubarev, V. V. et al. Threshold conditions for terahertz laser discharge in atmospheric gases // J. Infrared, Millimeter, and THz Waves. 2017. V 38, No. 6. P. 787-798.

7. Pavelyev, V. S. et al. Fabrication of high-effective silicon diffractive optics for the terahertz range by femtosecond laser ablation // Phys. Procedia. 2016. V. 84, P. 177-174.

8. Agafonov, A.N. et al. Focusing of Novosibirsk free electron laser (NovoFEL) radiation into paraxial segment // J. Modern Opt. 2016. V. 63. No. 11. P. 1051-1054.

9. Choporova, Y. Y. et al., High-power Bessel beams with orbital angular momentum in the terahertz range // Phys. Rev. A. 2017. Vol. 96. No. 2. 023846.

10. Knyazev, B., Serbo, V., Beams of photons with a nonzero projection of the orbital angular momentum - new results // Phys. Uspekhi, 2018. V. 61. No. 5. P. 449-479.

11. Knyazev B.A. et al. Quasi-Talbot effect with vortex beams and formation of vortex beamlet arrays // Opt. Expr. 2018. V. 26. No. 11. 14174.

12. Nikitin, P. A. et al. Deflection of terahertz vortex beam in nonpolar liquids by means of acousto-optics // Phys. Procedia (2016)

13. Kozlov, A.S. et al. Formation of Nanosized Metal Hydrosols under the Influence of Novosibirsk Terahertz free Electron Laser Radiation // Phys. Procedia. 2016. V. 84. P. 131-134.

14. Choporova, Yu. Yu. et al. Classical Holography in the Terahertz Range: Recording and Reconstruction Techniques // IEEE Trans. on THz Sci. Techn. 2015. V. 5, No. 5. P. 836-844.

15. Azarov, I. A. et al. Measurement of optical constants of liquids in the terahertz range using internal reflection ellipsometry // J. Infrared Millimeter THz Waves, 2018, submitted.

18. Gerasimov, V. V. et al. Growth of terahertz surface plasmon propagation length due to thin-layer dielectric coating // JOSA B. 2016. V. 33, No. 11. P. 2196-2203.

19. Gerasimov, V. V. et al. Wave-vector spectrum of monochromatic terahertz surface plasmon polaritons on real surfaces // Phys. Procedia. 2016. .V. 84. P. 157-164.

20. Knyazev, B. A. et al. Generation of terahertz surface plasmon polaritons using nondiffractive Bessel beams with orbital angular momentum // Phys. Rev. Lett. 2015. V. 115. No. 16. 163901.

21. Goryachkovskaya, T. N. et al. The impact of terahertz radiation on an extremophilic archaean Halorubrum saccharovorum proteome // Vavilov Journal of Genetics and Breeding. 2016. V. 20. No. 6. P. 869-875.

22. R. Kh. Zhukavin et al. Low-temperature intraceter relaxation times of shallow donors in germanium // JETP Lett. 2017. V. 106, No. 9, P. 571-575.

23. Veber, S. L. et al. X-band EPR setup with $\mathrm{THz}$ light excitation of Novosibirsk free electron laser: goals, means, useful extras // J. Magn. Res. 2018. V. 288. P. 11-22. 